\title{
Description of bulk inversion asymmetry in the effective-bond-orbital model
}

\author{
X. Cartoixà \\ T. J. Watson, Sr. Laboratories of Applied Physics 128-95, California Institute of Technology, Pasadena, California 91125, USA \\ D. Z.-Y. Ting \\ Jet Propulsion Laboratory, California Institute of Technology, Pasadena, California 91109, USA \\ T. C. McGill* \\ T. J. Watson, Sr. Laboratories of Applied Physics 128-95, California Institute of Technology, Pasadena, California 91125, USA
}

(Received 28 April 2003; published 12 December 2003)

\begin{abstract}
We have extended the effective-bond-orbital model (EBOM) method [Y. C. Chang, Phys. Rev. B 37, 8215 (1988)] to include the effects of the bulk inversion asymmetry (BIA) present in zinc blendes. This is accomplished without adding to the number of basis states or extending the range of interaction. We have also investigated a variant form of the EBOM proposed in the original formulation that offers improved zone-center behavior, but may also generate spurious solutions in heterostructure calculations due to poor description of bulk zone-boundary band structure. We offer suggestions for avoiding this problem so that this variant form of EBOM may be used safely. In general, we find that the addition of BIA effects in EBOM results in improved descriptions of zone-center band structure, but also in a loss of accuracy far from the Brillouin-zone center. We illustrate the use of the BIA extension with band-structure calculations for bulk GaSb. We show that the spin splitting predicted by the extended EBOM method for an AlSb/GaSb superlattice is in good agreement with $\mathbf{k} \cdot \mathbf{p}$ calculations that include BIA effects.
\end{abstract}

DOI: 10.1103/PhysRevB.68.235319

PACS number(s): 71.15.-m, 71.20.Nr, 73.21.-b

\section{INTRODUCTION}

The tight-binding method (TB) has been used extensively to compute band structures of bulk metals ${ }^{1-3}$ and semiconductors, ${ }^{1,4}$ and heterostructures, ${ }^{5,6}$ yielding a good compromise between accuracy and ease of implementation. It is a full zone method and, as such, has been used to describe situations where states corresponding to more than one extremum are needed, such as short period superlattices ${ }^{7}$ or $X$-point tunneling influence on the escape time of electrons inside leaky quantum wells. ${ }^{8}$

One of the difficulties tight-binding users encounter is the choice of material parameters. Usually, tight-binding parameters bear only an indirect relation with measurable quantities, and their determination normally requires a tedious fitting procedure. The effective-bond-orbital model (EBOM) method by Chang ${ }^{9,10}$ summarized in Sec. II provides a way of matching the TB parameters with the measurable $\mathbf{k} \cdot \mathbf{p}$ parameters using a reduced bond-orbital basis set ${ }^{11}$ located on a fcc lattice. This method is often used for studying semiconductor heterostructures. ${ }^{12}$ The limited number of bands and the focus on accurate band description near the zone center make it less adequate for full zone band features than the empirical TB or pseudopotential methods. ${ }^{13}$ On the other hand, because it is based on a full zone method, its validity range extends further into the Brillouin-zone boundary than its associated multiband $\mathbf{k} \cdot \mathbf{p}$, making it better suited than $\mathbf{k} \cdot \mathbf{p}$ for short period superlattices or narrow quantum wells.

Bulk inversion asymmetry (BIA) in III-V semiconductors refers to the lack of an inversion center in the zinc-blende structure. The time-reversal symmetry requires that a state $|\mathbf{k}, \uparrow\rangle$ be degenerate with a state $|-\mathbf{k}, \downarrow\rangle$. When this is combined with the inversion symmetry requirement that $|\mathbf{k}, \uparrow\rangle$ be degenerate with $|-\mathbf{k}, \uparrow\rangle$, the Kramers degeneracy condition that $E(\mathbf{k}, \uparrow)=E(\mathbf{k}, \downarrow)$ is obtained, and all bands are at least doubly degenerate. This is the case for group-IV elements such as diamond, $\mathrm{Si}, \mathrm{Ge}$, and $\alpha$-Sn. Since zinc blendes do not possess inversion symmetry, this allows for $E(\mathbf{k}, \uparrow)$ $\neq E(\mathbf{k}, \downarrow)$, which is of course realized only when the spinorbit interaction is included and the basis chosen for the band-structure computation reflects the inversion asymmetry (i.e., the basis states are not parity eigenstates).

As originally developed, the EBOM method does not account for the BIA present in zinc blendes, predicting doubly degenerate bands in structures where BIA lifts the degeneracy caused by the combination of time-reversal and inversion symmetry operations. ${ }^{14}$ In Sec. III, an extension to EBOM capable of describing BIA effects in the conduction band is presented. In Sec. IV the method is applied to bulk GaSb. Finally, Sec. V shows the application of the method to a symmetric $\mathrm{AlSb} / \mathrm{GaSb}$ superlattice, predicting the appearance of spin splitting in the conduction band. This is in contrast to standard $\mathbf{k} \cdot \mathbf{p}$ implementations or the original EBOM formulation, which would predict the absence of splitting. These results are shown to agree with $\mathbf{k} \cdot \mathbf{p}$ calculations that do account for BIA. ${ }^{15}$

\section{THE EBOM METHOD}

The basic idea of the EBOM method is to take the TB Hamiltonian expressed in a bond-orbital model basis set, series expand it for small $k$ 's, and then compare the matrix elements with the $\mathbf{k} \cdot \mathbf{p}$ matrix elements ${ }^{16}$ to obtain the TB parameters in terms of the $\mathbf{k} \cdot \mathbf{p}$ parameters.

Following Chang, ${ }^{9}$ the orbitals are located at points of a fcc lattice. A state at a site $\mathbf{R}$ with $\alpha=s, x, y, z$ character is 
labeled $|\mathbf{R}, \alpha\rangle$. The bond orbitals are taken to be the Löwdin symmetrized orbitals ${ }^{17}$ that most closely resemble the top of the valence and bottom of the conduction-band states. The requirement that they originate from linear combination of atomic orbitals in a unit cell does not need to be made. The success of the method (and of the $\mathbf{k} \cdot \mathbf{p}$ method as well) comes precisely from the fact that the exact form of the wave functions is not needed to compute the Hamiltonian matrix elements. Instead, these matrix elements are considered empirical parameters ${ }^{1,18}$ to be fitted to experimental data or to more accurate band structures obtained by computationally more expensive methods.

The interaction between the $p$-type orbitals $|\mathbf{R}, \beta\rangle$ and $\left|\mathbf{R}^{\prime}, \beta^{\prime}\right\rangle(\beta=x, y, z)$ for the fcc lattice is given by ${ }^{10}$

$$
\begin{aligned}
\left\langle\mathbf{R}, \beta|H| \mathbf{R}^{\prime}, \beta^{\prime}\right\rangle= & E_{p} \delta_{R, R^{\prime}} \delta_{\beta, \beta^{\prime}} \\
& +\sum_{\tau} \delta_{R^{\prime}-R, \tau}\left\{E_{x y} \tau_{\beta} \tau_{\beta^{\prime}}\left(1-\delta_{\beta, \beta^{\prime}}\right)\right. \\
& \left.+\left[E_{x x} \tau_{\beta}^{2}+E_{z z}\left(1-\tau_{\beta}^{2}\right)\right] \delta_{\beta, \beta^{\prime}}\right\},
\end{aligned}
$$

where $E_{p}$ is the on-site energy and $E_{x y}, E_{x x}$, and $E_{z z}$ are different nearest-neighbor interaction parameters. The vectors $\tau$ join the $12 \mathrm{fcc}$ nearest neighbors, and they can have values

$$
\tau=\frac{a}{2}([ \pm 1, \pm 1,0],[ \pm 1,0, \pm 1],[0, \pm 1, \pm 1]),
$$

with $a$ being the lattice constant.

The interaction of $s$ orbitals is simply

$$
\left\langle\mathbf{R}, s|H| \mathbf{R}^{\prime}, s\right\rangle=E_{s} \delta_{R, R^{\prime}}+\sum_{\tau} E_{s s} \delta_{R^{\prime}-R, \tau},
$$

with $E_{s}$ and $E_{s s}$ being the on-site and the nearest-neighbor interaction parameters, respectively. The remaining interaction is between the $s$ - and $p$-like orbitals at nearest-neighbor sites:

$$
\left\langle\mathbf{R}, s|H| \mathbf{R}^{\prime}, \beta\right\rangle=E_{s x} \tau_{\beta} \delta_{R^{\prime}-R, \tau} .
$$

At this point, a clarification must be made. The lack of inversion asymmetry in the ordinary EBOM method and its inability to describe the reduced $T_{d}$ symmetry of zinc blendes does not originate from the basis set being located on a fcc lattice, but rather on the fact that a basis with a definite parity has been used to obtain Eqs. (1)-(4). In the following section this assumption is relaxed, yielding the correction necessary to describe spin splitting in the conduction band.

From the Löwdin functions $|\mathbf{R}, \alpha\rangle$, Bloch sums can be written in the form

$$
|\mathbf{k}, \alpha\rangle=\frac{1}{\sqrt{N}} \sum_{\mathbf{R}} e^{i \mathbf{k} \cdot \mathbf{R}}|\mathbf{R}, \alpha\rangle,
$$

where $N$ is the number of unit cells in the sample. Each eigenstate with a wave vector $\mathbf{k}$ is written as a linear combination of Bloch sums: ${ }^{19}$

$$
\left|\Psi_{\mathbf{k}}\right\rangle=\sum_{\alpha} u_{\alpha}|\mathbf{k}, \alpha\rangle
$$

The coefficients $u_{\alpha}$ are found by seeking stationary values of $\left\langle\Psi_{\mathbf{k}}|H| \Psi_{\mathbf{k}}\right\rangle /\left\langle\Psi_{\mathbf{k}} \mid \Psi_{\mathbf{k}}\right\rangle$, which leads to the diagonalization of a Hamiltonian with matrix elements

$$
\begin{aligned}
\left\langle\mathbf{k}, \alpha|H| \mathbf{k}, \alpha^{\prime}\right\rangle & =\frac{1}{N} \sum_{\mathbf{R}, \mathbf{R}^{\prime}} e^{i \mathbf{k} \cdot\left(\mathbf{R}^{\prime}-\mathbf{R}\right)}\left\langle\mathbf{R}, \alpha|H| \mathbf{R}^{\prime}, \alpha^{\prime}\right\rangle \\
& =\sum_{\mathbf{R}^{\prime}} e^{i \mathbf{k} \cdot \mathbf{R}^{\prime}}\left\langle\mathbf{R}=0, \alpha|H| \mathbf{R}^{\prime}, \alpha^{\prime}\right\rangle .
\end{aligned}
$$

For the $p$ sub-block, substituting Eq. (1) into Eq. (7) yields

$$
\begin{aligned}
H_{\beta, \beta^{\prime}}(\mathbf{k})= & E_{p} \delta_{\beta, \beta^{\prime}}+\sum_{\tau} e^{i \mathbf{k} \cdot \tau}\left\{E_{x y} \tau_{\beta} \tau_{\beta^{\prime}}\left(1-\delta_{\beta, \beta^{\prime}}\right)\right. \\
& \left.+\left[E_{x x} \tau_{\beta}^{2}+E_{z z}\left(1-\tau_{\beta}^{2}\right)\right] \delta_{\beta, \beta^{\prime}}\right\} .
\end{aligned}
$$

Similarly, it is easy to see that

$$
H_{s, s}(\mathbf{k})=E_{s}+\sum_{\tau} e^{i \mathbf{k} \cdot \tau} E_{s s}
$$

and

$$
H_{s, \beta}(\mathbf{k})=\sum_{\tau} e^{i \mathbf{k} \cdot \tau} E_{s x} \tau_{\beta}
$$

In order to find values for the EBOM parameters, the sums over first neighbors in Eqs. (8)-(10) are evaluated. For example, it is easy to see that

$$
H_{s, x}=4 i E_{s x} \sin \xi(\cos \eta+\cos \zeta),
$$

where $\xi=k_{x} a / 2, \eta=k_{y} a / 2$, and $\zeta=k_{z} a / 2$. This agrees with the value in Table II of Ref. 1, provided that terms occupied in the simple cubic but not in the fcc lattices are disregarded.

Then, the matrix elements are series expanded up to second order in $\mathbf{k}$ and compared to $\mathbf{k} \cdot \mathbf{p}$ matrix elements ${ }^{18}$ to obtain the relations listed in Table I. The values of the $\mathbf{k} \cdot \mathbf{p}$ parameters $L^{\prime}, N^{\prime}$ in terms of the more common $L, N$ are available, for example, Eq. (13) in Ref. 20. Note that the last entry in that table is not totally determined. Taking $E_{s x}$ $=P / 4 a$ might seem the sensible thing to do, but it produces spurious solutions. ${ }^{9}$ Instead, the auxiliary constraint $\left(E_{z z}\right.$ $\left.-E_{x x}\right)=X_{h l} / 8$, where $X_{h l}$ is the heavy-hole-light-hole separation at the $X$ point, is used. Setting $X_{h l}$ at the $X$ point allows the condition $E_{s x}=P / 4 a$ to be approximately satisfied for narrow-gap materials. ${ }^{9}$

Spin-orbit effects have been introduced in the TB model by Chadi. ${ }^{21}$ In the EBOM method, they are simply introduced by adding spin to the basis states, performing a change of basis on the Hamiltonian into a $|j, m\rangle$ basis, and then modifying the diagonal components of the energies to include the spin-orbit splitting. This procedure reproduces, by construction, the spin-orbit (SO) splitting at the $\Gamma$ point. In the case of $\mathrm{GaSb}$, it predicts a SO splitting at the $L$ point $\Delta_{1}$ of $0.49 \mathrm{eV}$, vs an experimental value ${ }^{22}$ of $0.45 \mathrm{eV}$, while 
TABLE I. Relationship between the EBOM parameters and the $\mathbf{k} \cdot \mathbf{p}$ parameters.

Parameters

$$
\begin{aligned}
& E_{s}=E_{c}+12 \frac{A^{\prime}+\hbar^{2} / 2 m_{0}}{a^{2}} \\
& E_{s s}=-\frac{A^{\prime}+\hbar^{2} / 2 m_{0}}{a^{2}} \\
& E_{p}=E_{v}+2 \frac{3 \hbar^{2} / 2 m_{0}+2 L^{\prime}+4 M}{a^{2}} \\
& E_{x x}=-\frac{\hbar^{2} / 2 m_{0}+2 L^{\prime}}{2 a^{2}} \\
& E_{z z}=-\frac{\hbar^{2} / 2 m_{0}-2 L^{\prime}+4 M}{2 a^{2}} \\
& E_{x y}=E_{x y}(110)=-\frac{N^{\prime}}{a^{2}}
\end{aligned}
$$

(a) $E_{s x}=\frac{P}{4 a}$ or (b) $\left(E_{z z}-E_{x x}\right)=X_{h l} / 8=0.5 \mathrm{eV}$

overestimating $X_{7 v}-X_{6 v}$ by a factor of 2 with respect to the nonlocal pseudopotential value. ${ }^{13}$

\section{INCLUSION OF BULK INVERSION ASYMMETRY EFFECTS IN EBOM}

As previously indicated, the EBOM Hamiltonian in zinc blendes reproduces an $O_{h}$ point group symmetry rather than the reduced $T_{d}$ because the basis states are implicitly assumed to be parity eigenstates. Specifically, the $p$ states, which are the strongest components in valence-band states, have negative parity. The simplest way to introduce an inversion symmetry-breaking component consistent with the $\Gamma_{5}$ symmetry $^{32}$ of the valence states is to add some $d$ character to the $p$ states. The reason for this is that $d$ states are close energetically to $p$ states, and a finite $d$ contribution to top of the valence-bend(VB) states is found indeed in $s p d s^{*} \mathrm{~TB}$ calculations (see, for example, Fig. 4 in Ref. 4, where the $d$ contribution to the top of the VB is $\approx 20 \%$ ). Thus, the substitutions

$$
\begin{gathered}
|\mathbf{R}, x\rangle \rightarrow c_{p}|\mathbf{R}, x\rangle+c_{d}|\mathbf{R}, y z\rangle, \\
|\mathbf{R}, y\rangle \rightarrow c_{p}|\mathbf{R}, y\rangle+c_{d}|\mathbf{R}, z x\rangle, \\
|\mathbf{R}, z\rangle \rightarrow c_{p}|\mathbf{R}, z\rangle+c_{d}|\mathbf{R}, x y\rangle,
\end{gathered}
$$

are made, where $c_{p}$ and $c_{d}$ are real constants that measure the importance of the odd and even (under inversion) components in the new state, respectively.

Using the matrix element $H_{s, x}$ as an example, the change in the states will transform it to

$$
H_{s, x}=c_{p}\langle\mathbf{k}, s|H| \mathbf{k}, x\rangle+c_{d}\langle\mathbf{k}, s|H| \mathbf{k}, y z\rangle .
$$

Looking up again in Table II of Ref. 1, one can see that

$$
H_{s, x}=4 i E_{s x} \sin \xi(\cos \eta+\cos \zeta)-4 E_{s, x y} \sin \eta \sin \zeta,
$$

where the coefficients $c_{i}$ have been absorbed into the adjustable parameters $E_{i}$. Now, comparing Eq. (14) with the corresponding element in the $\mathbf{k} \cdot \mathbf{p}$ Hamiltonian in Ref. 18,

$$
H_{s x, \mathbf{k} \cdot \mathbf{p}}=i P k_{x}+B k_{y} k_{z},
$$

one sees that the parameter $B$ (Ref. 18)

$$
B=2 \frac{\hbar^{2}}{m^{2}} \sum_{j}^{\Gamma_{15}^{\ddagger V B}} \frac{\left\langle s\left|p_{x}\right| u_{j}\right\rangle\left\langle u_{j}\left|p_{y}\right| z\right\rangle}{\left\{\left[\left(E_{c}+E_{v}\right) / 2\right]-E_{j}\right\}}
$$

describing the BIA in the $\mathbf{k} \cdot \mathbf{p}$ formalism can be introduced in EBOM by taking

$$
E_{s, x y}=-B / a^{2}
$$

Therefore, the inclusion of BIA is made at a negligible computational cost and its implementation is straightforward because we are only adding a supplemental matrix element. On the other hand, describing BIA and its associated spin splitting by using anion and cation orbitals and on-site spinorbit matrix elements ${ }^{21}$ would require extending the dimensionality of the basis set. An additional property of the present method is that the number of neighbors included in the calculation is not increased.

It remains to be seen how the remaining matrix elements are affected by the substitutions (12). $H_{s, s}$ is left unchanged, while the other diagonal elements become

$$
\begin{aligned}
H_{x, x}= & {\left[\left|c_{p}\right|^{2} 4 E_{x x}+\left|c_{d}\right|^{2} 4 E_{x y, x y}(011)\right](\cos \xi \cos \eta} \\
& +\cos \xi \cos \zeta)+\left[\left|c_{p}\right|^{2} 4 E_{z z}\right. \\
& \left.+\left|c_{d}\right|^{2} 4 E_{x y, x y}(110)\right] \cos \eta \cos \zeta \\
= & 4 E_{x x}(\cos \xi \cos \eta+\cos \xi \cos \zeta)+4 E_{z z} \cos \eta \cos \zeta,
\end{aligned}
$$

where in the last step $E_{x x}$ and $E_{z z}$ have been redefined so that Table I still holds. The other diagonal elements can be obtained by the appropriate cyclic permutations.

The nondiagonal elements between $\Gamma_{5}$ states also change:

$$
\begin{aligned}
H_{x, y}= & -4\left[\left|c_{p}\right|^{2} E_{x y}+\left|c_{d}\right|^{2} E_{x y, x z}(011)\right] \sin \xi \sin \eta \\
& -4 i c_{p} c_{d}\left[E_{x, x y}(011)\right. \\
& \left.-E_{x, x y}(110)\right] \sin \zeta[\cos \xi-\cos \eta] \\
= & -4 E_{x y}(110) \sin \xi \sin \eta \\
& -4 i E_{x y}(011) \sin \zeta[\cos \xi-\cos \eta],
\end{aligned}
$$

with the usual redefinition of parameters in the last step. $H_{y, z}$ and $H_{z, x}$ are obtained by cyclic permutations. The results here obtained for the tight-binding zinc-blende matrix elements agree with those of Hass et al., ${ }^{23}$ which correct the misprints in Table V of Ref. 1.

Comparison with the $\mathbf{k} \cdot \mathbf{p}$ Hamiltonian does not provide the value of the $E_{x y}(011)$ parameter because it only introduces terms of order $k^{3}$ or higher when the corresponding matrix element is expanded. This should not be a concern when we seek only properties of states near the $\Gamma$ point. A 
a)

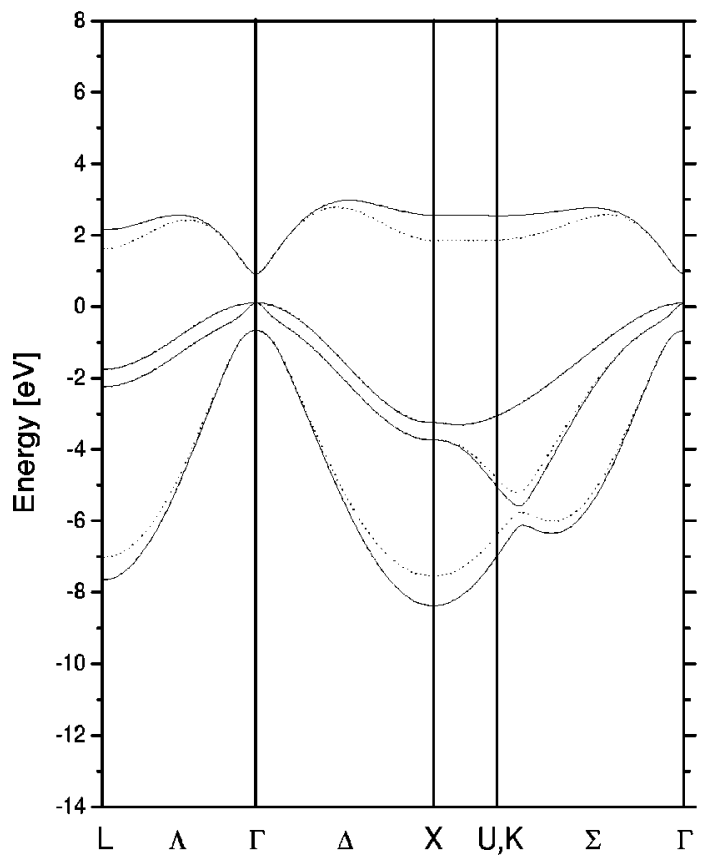

b)

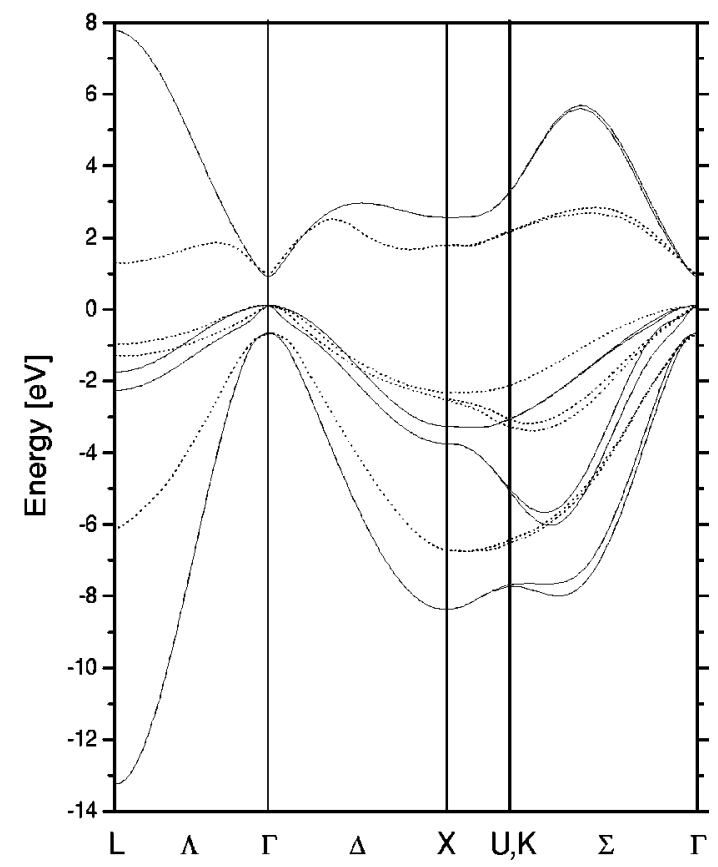

FIG. 1. Band structure of GaSb calculated with EBOM under different assumptions for the parameters. The dotted line in plot (a) is obtained under the original requirement (Ref. 9) that the separation $X_{h l}=4.0 \mathrm{eV}$ is used to obtain $E_{s x}$. The solid line is obtained taking $E_{s x}=P / 4 a$. A term describing BIA has been included in the solid line for plot (b), which otherwise uses the same set of parameters as in the calculations represented by the solid line in plot (a). The spin split bands along the $\Sigma$ line can be observed. The dotted lines in plot (b) are results from nonlocal pseudopotential calculations from Ref. 13 shown for comparison.

look at the matrix elements reveals that the contribution of $E_{x y}(011)$ reaches its peak near the $\mathrm{K}$ point. Since we are only interested in properties near the zone center, its value will be set to zero for the following calculations.

\section{BULK BAND STRUCTURE}

The considerations above are illustrated with a sample calculation of bulk GaSb. Close to the zone center of a zinc blende, spin-orbit causes cubic splitting of the conduction and the SO bands ${ }^{24,15}$ along the [110] direction. It also causes a linear splitting of the heavy-hole $(\mathrm{HH})$ and light-hole $(\mathrm{LH})$ bands along [110], a linear splitting of only the HH bands along [111] and makes both the $\mathrm{HH}$ and $\mathrm{LH}$ have a finite slope along [100] while keeping the double degeneracy. ${ }^{24}$

Figure 1 shows bulk GaSb band structures calculated using EBOM, both with and without BIA effects, and compared to pseudopotential calculations. The solid and dotted lines in plot (a) correspond to the EBOM model without the zinc blende symmetry corrections. The dotted line is obtained under the original requirement ${ }^{9}$ that the separation $X_{h l}=4.0 \mathrm{eV}$ is used to obtain $E_{s x}$. We will call this the $X$ model. The solid line is obtained taking the alternative prescription ${ }^{9} E_{s x}=P / 4 a$, with $P$ obtained from the value of the effective mass. This will be called the $P$ model. With the parameters used, looking at the $X$ and $L$ points, in the $P$ model the conduction band (CB) and split-off (SO) band are pushed further away than in the $X$ model.

Although the $P$ model could in principle provide a more accurate zone-center band-structure description, it should be used with some caution. In the $P$ model, the position of the SO band at the $X$ point is very sensitive to the value of the $\mathrm{CB}$ effective mass. For example, changing $m_{c}^{*} / m_{0}$ - where $m_{0}$ is the free-electron mass-in InAs from 0.025 to 0.024 changes the position of the $\mathrm{SO}$ band from about $-10 \mathrm{eV}$ to about $-6.5 \mathrm{eV}$. Going one step further, as illustrated in Fig. 2 , setting $m_{c}^{*}=0.023 m_{0}$ causes the SO band to anticross with the light-hole band at some point along the $\Delta$ line, and the spurious valence-band crossing described in Ref. 9 appears. All these values compare favorably with the experimental value $^{25} m_{c, \text { InAs }}^{*}=0.024 m_{0}$. Therefore, it is reasonable to assume that very small changes in the value of the $m_{c}^{*} / m_{0}$ parameter can get rid of spurious solutions present in the $P$ model for some materials. In particular, this avoids the case where the LH band would create an $X$ valley in the gap region that might originate spurious superlattice bands. ${ }^{26} \mathrm{~A}$ good procedure to avoid these complications would be to create a plot as in Fig. 2 for each of the constituent bulk materials, and to make sure that the LH-HH crossing does not take place.

The solid line for plot (b) in Fig. 1 is generated under the same conditions as model $P$, but with BIA effects turned on by letting $E_{s, x y}=-B / a^{2}$. This will be called the $P B$ model. In agreement with predictions from the character tables for the $T_{d}$ group, ${ }^{27}$ the bands become spin split in the $\Sigma$ direction because of the breakdown of Kramers degeneracy. However, the correct description of the zinc-blende symmetry is 


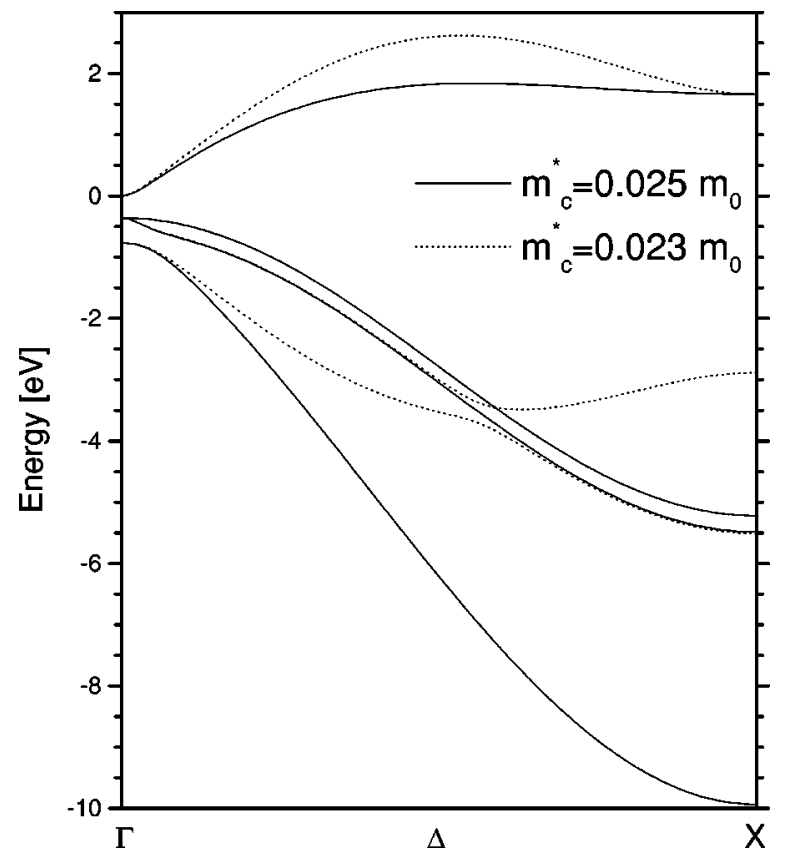

FIG. 2. Bands for bulk InAs along the $\Delta$ line. It is observed how a slight change of the value of the conduction-band effective mass, while keeping the rest of the parameters constant, induces the appearance of a spurious crossing of the LH and HH bands (dotted line).

made at the cost of the loss of accuracy for the CB and SO bands, specially along the $\Lambda$ line, where they take values quite far from pseudopotential calculations, ${ }^{13}$ represented by the dotted line. The inclusion of a finite $B$ in the EBOM calculations does not change substantially the $\mathrm{HH}$ and $\mathrm{LH}$ bands. The preference of having a correct description of the bands near the $\Gamma$ point or the $\Delta$ line including spin—with its ability to describe short period (100) superlattices-or a more accurate full zone description will determine the model to be used. The inclusion of second nearest-neighbor matrix elements ${ }^{28}$ might reconcile the energy values at the $L$ point in the $P B$ model with the pseudopotential calculations and experimental findings. ${ }^{22}$

Figure 3(a) shows the bands in more detail close to the zone center, with the spin splitting in the bands along the [110] direction. Plot (b) shows the splitting $\mathcal{S}$ in the conduction band along the $\Sigma$ line for the $\mathrm{CB}$, and a fit using

$$
\mathcal{S}=\gamma_{c} k^{3},
$$

where $\gamma_{c}$ is the $k^{3}$ splitting proportionality constant. The value used for $\gamma_{c}$ is $186 \mathrm{eV} \AA^{3}$, in good agreement with the measured $^{29}$ value of $187 \mathrm{eV} \AA^{3}$. This shows that the parameter $B$ determines the $\mathrm{CB}$ splitting near the zone center in the $P B$ model in the same way as it does in the $\mathbf{k} \cdot \mathbf{p}$ method, as expected from the derivation in Sec. III. A look at Fig. 3(b) reveals that, for GaSb, expression (20) is good until about $2 \%$ of the zone edge. Figure $3(\mathrm{c})$ shows that, as expected, ${ }^{24}$ the behavior of the splitting for the SO band is described by $\gamma_{S O} k^{3}$ close to the zone center. The range of validity of the cubic expression is extended with respect to the CB case. In our calculation we find that $\gamma_{S O}=196 \mathrm{eV}^{3}$. We are not
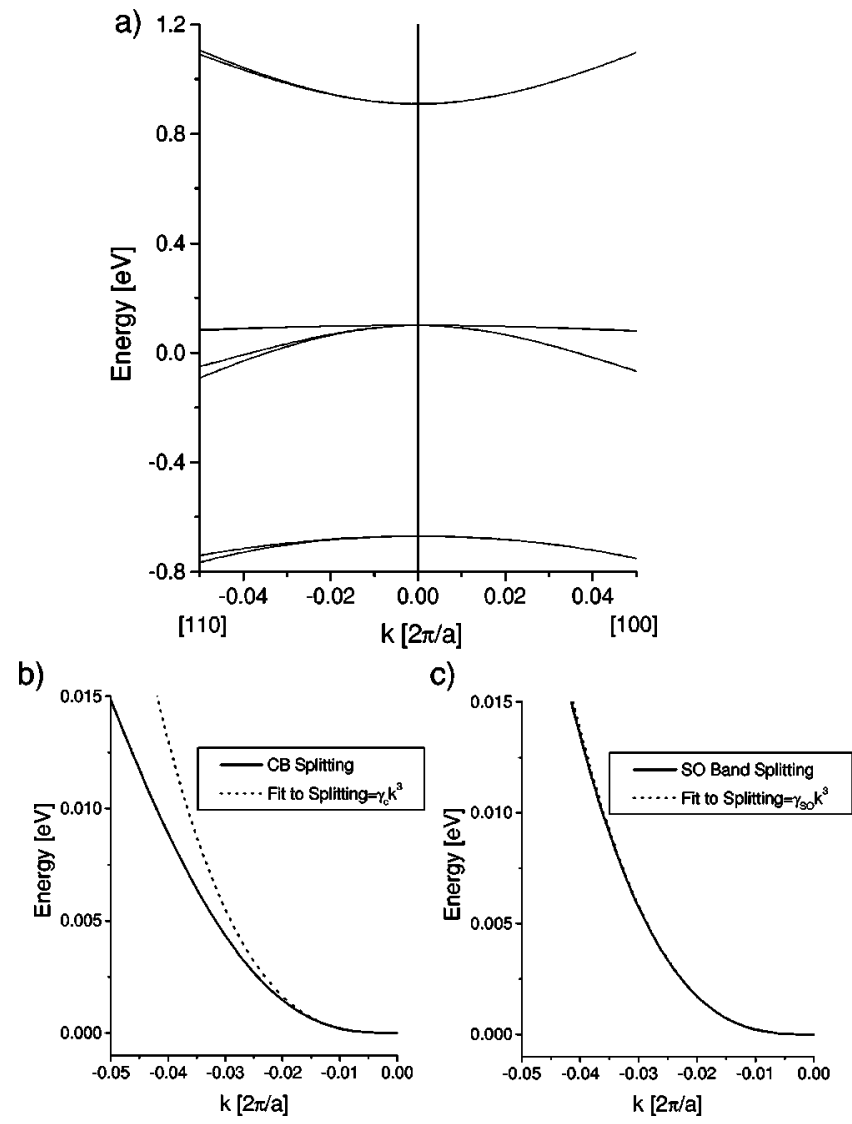

FIG. 3. (a) Bands close to the zone center showing the spin splitting, calculated with EBOM. (b) Amount of CB splitting and its $k^{3}$ dependence at low values of $k$. (c) As in (b), but with respect to the SO band.

aware of any experimental measurement of $\gamma_{S O}$, but this result is of the same order of magnitude as the calculations by Cardona et al. ${ }^{30}$

The only qualitative aspect of the bulk bands that the extension in Sec. III cannot incorporate is the linear spin splitting in the valence bands close to the zone center. ${ }^{24} \mathrm{In}$ $\mathbf{k} \cdot \mathbf{p}$, this is described by a parameter $C$ coming from secondorder mixed $\mathbf{k} \cdot \mathbf{p}$ and spin-orbit terms in the perturbation expansion. ${ }^{18,30}$ It can be seen from the LH bands plotted in Fig. 3 that the splitting that we obtain for that band is cubic in $k$-the splitting for the $\mathrm{HH}$ bands is also cubic in $k$-while it should be linear for both bands if the effects of $C$ were properly described in our extension. In the tightbinding method it is possible to include the effects of both the $B$ parameter ${ }^{21}$ and the $C$ parameter ${ }^{31}$ using additional matrix elements defined in the anion-cation basis. However, the reduced EBOM basis set makes it difficult to include the effects of $C$ in a straightforward manner. In any case, the effects of $C$ are normally small, and its importance for heterostructures is studied, for a particular case, in the following section.

\section{BULK INVERSION ASYMMETRY EFFECTS IN SYMMETRIC SUPERLATTICES}

The extension of the EBOM method in Sec. III is tested with the calculation of the band structure of a symmetric 


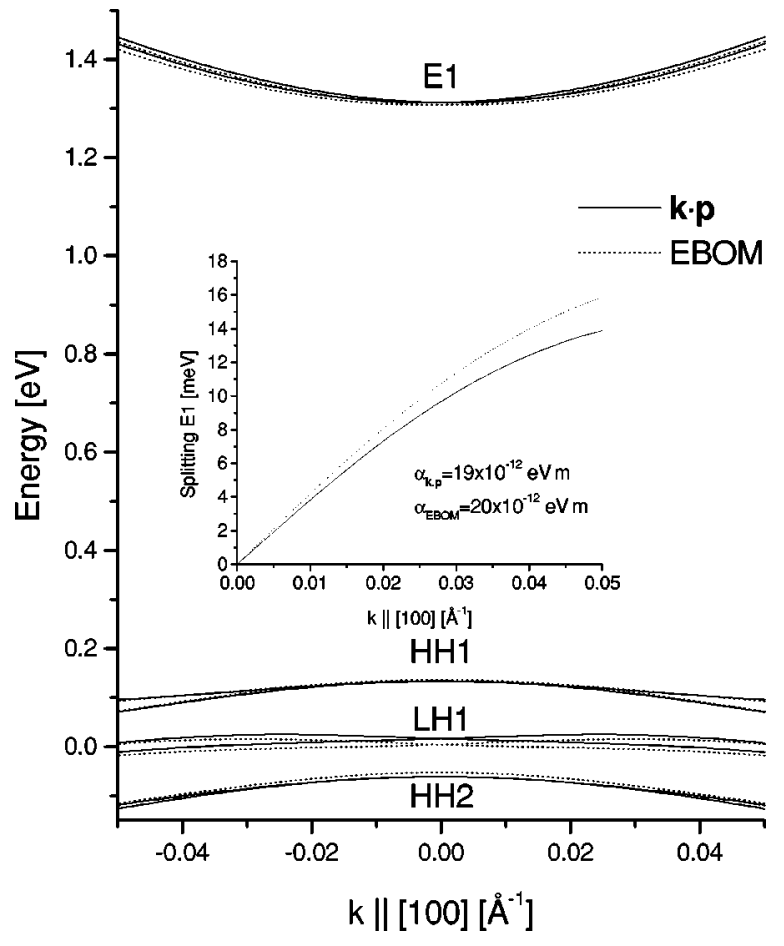

FIG. 4. Comparison of EBOM and $\mathbf{k} \cdot \mathbf{p}$ superlattice bands. The structure is a $16 / 8 \mathrm{AlSb} / \mathrm{GaSb} \mathrm{SL}$. The solid (dotted) lines are the $\mathbf{k} \cdot \mathbf{p}(\mathrm{EBOM})$ results. The bands are spin split away from $\Gamma$ due to the bulk inversion asymmetry. The inset shows, for both methods, the amount of splitting in the E1 band and the values for the splitting coefficients as defined by $\Delta E=2 \alpha k$.

$\mathrm{AlSb} / \mathrm{GaSb}$ superlattice (SL). The reduction of the symmetry due to the confinement causes the states in the $\mathrm{CB}$ to become spin split even along the [100] direction, ${ }^{14}$ in contrast to the predictions of $\mathbf{k} \cdot \mathbf{p}$ implementations that do not include BIA effects.

Figure 4 shows the comparison of the bands of a $16 / 8$ $\mathrm{AlSb} / \mathrm{GaSb} \mathrm{SL}$ calculated by both the $\mathbf{k} \cdot \mathbf{p}$ and the EBOM methods. In this $\mathbf{k} \cdot \mathbf{p}$ calculation, which does include BIA effects, ${ }^{15}$ the parameters $B$ and $C$ describing BIA are both set to finite values for $\mathrm{GaSb}$. In the EBOM calculation, as stated previously, only $B$ can be set. Control calculations have been performed using $\mathbf{k} \cdot \mathbf{p}$ with $C=0$ and $C \neq 0$ for this structure and for a $16 / 8 / 8 \mathrm{AlSb} / \mathrm{GaSb} / \mathrm{InAs} \mathrm{SL}$, and we always found that the inclusion of $C$ modified the splittings only by a few tenths of meV. Thus, at least for this system, the inability of the $P B$ model to describe the linear splitting in the valence bands of bulk zinc blendes does not constitute a serious drawback when studying splittings in heterostructures. The solid (dotted) lines correspond to the $\mathbf{k} \cdot \mathbf{p}(\mathrm{EBOM})$ results. It can be seen that the E1, HH1, and $\mathrm{HH} 2$ levels are slightly closer to the bulk band edge levels due to nonparabolicity effects. This not being the case for the LH1 band might be due to different boundary conditions, strain implementations, etc. In the inset, the amount of splitting between the E1 subbands is shown, with both methods yielding similar results.

\section{CONCLUSIONS}

In conclusion, an extension to Chang's EBOM method ${ }^{9}$ for obtaining band structures has been presented. This extension can describe the cubic spin splitting in the bulk zincblende conduction bands, but cannot describe the linear splitting present in bulk hole bands, where a cubic spin splitting is incorrectly obtained. However, the lack of inclusion of this linear splitting does not have appreciable consequences for the subbands of the heterostructure we have studied. The inclusion of the bulk inversion asymmetry effects is made at the cost of a loss of accuracy for the bulk bands at some points far from the Brillouin-zone center. Also, we have reexamined the issue of spurious solutions originally discussed by Chang ${ }^{9}$ in the context of an alternative EBOM formulation that does not constrain the HH-LH separation at the $X$ point. We have shown that the spurious solutions can be eliminated with small changes in the value of the conduction-band effective mass. To use this variant of EBOM for heterostructure calculation, we recommend a careful screening of the bulk band structures of all constituent materials for anomalous behavior along the $\Delta$ direction-and fixing the problem by making small adjustments in conduction-band effective mass if necessary; this will prevent the appearance of spurious solutions arising from folded $X$ point states associated with erroneous bulk bands. Finally, the method has been applied to the calculation of bulk GaSb and an AlSb/GaSb superlattice, and shown to have good agreement close to the zone center with results from $\mathbf{k} \cdot \mathbf{p}$ calculations that include BIA effects.

\section{ACKNOWLEDGMENTS}

The authors would like to thank Y.-C. Chang for helpful discussions. This work has been supported by the Office of Naval Research under Grant No. N00014-98-1-0567. A part of this work was carried out at the Jet Propulsion Laboratory, California Institute of Technology, and was sponsored by the Defense Advanced Research Projects Agency SpinS program through an agreement with the National Aeronautics and Space Administration.
*Electronic address: tcm@ssdp.caltech.edu.

${ }^{1}$ J.C. Slater and G.F. Koster, Phys. Rev. 94, 1498 (1954).

${ }^{2}$ M. Asdente and J. Friedel, Phys. Rev. 124, 384 (1961).

${ }^{3}$ M.J. Mehl and D.A. Papaconstantopoulos, Phys. Rev. B 54, 4519 (1996).

${ }^{4}$ J.M. Jancu, R. Scholz, F. Beltram, and F. Bassani, Phys. Rev. B 57, 6493 (1998).

${ }^{5}$ J.N. Schulman and Y.C. Chang, Phys. Rev. B 24, 4445 (1981).
${ }^{6}$ J.N. Schulman and Y.C. Chang, Phys. Rev. B 27, 2346 (1983).

${ }^{7}$ T. Matsuoka, T. Nakazawa, T. Ohya, K. Taniguchi, C. Hamaguchi, H. Kato, and Y. Watanabe, Phys. Rev. B 43, 11798 (1991).

${ }^{8}$ M.K. Jackson, D. Ting, D.H. Chow, D.A. Collins, J.R. Soderstrom, and T.C. McGill, Phys. Rev. B 43, 4856 (1991).

${ }^{9}$ Y.C. Chang, Phys. Rev. B 37, 8215 (1988).

${ }^{10}$ G.T. Einevoll and Y.C. Chang, Phys. Rev. B 40, 9683 (1989).

${ }^{11}$ W.A. Harrison, Phys. Rev. B 8, 4487 (1973). 
${ }^{12}$ D.N. Talwar, J.P. Loehr, and B. Jogai, Phys. Rev. B 49, 10345 (1994)

${ }^{13}$ J.R. Chelikowsky and M.L. Cohen, Phys. Rev. B 14, 556 (1976).

${ }^{14}$ R. Eppenga and M.F.H. Schuurmans, Phys. Rev. B 37, 10923 (1988).

${ }^{15}$ X. Cartoixà, D.Z.-Y. Ting, and T.C. McGill, cond-mat/0212394 (unpublished).

${ }^{16}$ E.O. Kane, J. Phys. Chem. Solids 1, 82 (1956).

${ }^{17}$ P.-O. Löwdin, J. Chem. Phys. 18, 365 (1950).

${ }^{18}$ E.O. Kane, in Semiconductors and Semimetals, edited by R.K. Willardson and A.C. Beer (Academic, New York, 1966), Vol. 1, pp. 75-100.

${ }^{19}$ W.A. Harrison, Electronic Structure and the Properties of Solids, 1st ed. (Dover, Mineola, 1989).

${ }^{20}$ R. Enderlein, G.M. Sipahi, L.M.R. Scolfaro, and J.R. Leite, Phys. Status Solidi B 206, 623 (1998).

${ }^{21}$ D.J. Chadi, Phys. Rev. B 16, 790 (1977).

${ }^{22}$ T.C. Chiang and D.E. Eastman, Phys. Rev. B 22, 2940 (1980).
${ }^{23}$ K.C. Hass, H. Ehrenreich, and B. Velicky, Phys. Rev. B 27, 1088 (1983).

${ }^{24}$ G. Dresselhaus, Phys. Rev. 100, 580 (1955).

${ }^{25}$ C.R. Pidgeon, D.L. Mitchell, and R.N. Brown, Phys. Rev. 154, 737 (1967).

${ }^{26}$ X. Cartoixà, D.Z.-Y. Ting, and T.C. McGill, J. Appl. Phys. 93, 3974 (2003).

${ }^{27}$ G.F. Koster, J.O. Dimmock, R.G. Wheeler, and H. Statz, Properties of the Thirty-Two Point Groups, 1st ed. (MIT Press, Cambridge, MA, 1963).

${ }^{28}$ J.P. Loehr, Phys. Rev. B 50, 5429 (1994).

${ }^{29}$ G.E. Pikus, V.A. Marushchak, and A.N. Titkov, Fiz. Tekhn. Poluprovodn. 22, 185 (1988) [Sov. Phys. Semicond. 22, 115 (1988)].

${ }^{30}$ M. Cardona, N.E. Christensen, and G. Fasol, Phys. Rev. B 38, 1806 (1988).

${ }^{31}$ T.B. Boykin, Phys. Rev. B 57, 1620 (1998).

${ }^{32}$ The KDWS notation is being used (see Ref. 27). 